\title{
BROWNIAN MOTION OF A PLASMA CRYSTAL
}

\author{
A. Ignatov \\ Prokhorov General Physics Institute, Russian Academy of Sciences, \\ 119991, Moscow, Vavilov Str., 38, Russia
}

We discuss the dynamics of a plasma crystal under the influence of random forces. The set of Langevin equations is solved in harmonic approximation. Pair correlation function of particle displacements are expressed in terms of phononic spectra.

As an example, we study the hexagonal single-layer plasma crystal. It is shown that singular points of wave dispersion curves manifest themselves as characteristic patterns in pair correlation spectra. 